\title{
BONE MINERAL DENSITY AND TRABECULAR BONE SCORE IN POSTMENOPAUSAL WOMEN WITH KNEE OSTEOARTHRITIS AND OBESITY
}

DOI: 10.36740/WLek202003124

\author{
Vladyslav V. Povoroznyuk ${ }^{2}$, Nataliia V. Zaverukha ${ }^{1,2}$, Anna S. Musiienko ${ }^{2}$ \\ 'SHUPYK NATIONAL MEDICAL ACADEMY OF POSTGRADUATE EDUCATION, KYIV, UKRAINE \\ ${ }^{2}$ D. F. CHEBOTAREV INSTITUTE OF GERONTOLOGY, NAMS OF UKRAINE, KYIV, UKRAINE
}

\begin{abstract}
The aim of the study was to investigate the relationship between bone mineral density (BMD) of lumbar spine, femoral neck, trabecular bone score (TBS) and body mass index (BMI), in postmenopausal women with knee osteoarthritis (OA).

Materials and methods: The study group comprised 359 postmenopausal women aged 50-89 years. They were divided into 2 groups: I group - 117 postmenopausal women with symptomatic knee $\mathrm{OA}$ and II group -242 women with a normal functional activity of knee joints. Analysis of data was performed taking into account their BMD of lumbar spine (L1-L4) and femoral neck, measured by the Dual-energy X-ray absorptiometry (DXA) Hologic (Discovery WI, USA, 2016). TBS of L1-L4 was detected by TBS insight ${ }^{\circledR}$ software (Medlmaps, Pessac, France), and BMI classified by World Health Organization (WHO).

Results: In postmenopausal women with obesity prevalence of symptomatic knee 0A was detected in $41.1 \%$ of cases. However, in women with normal BMI knee 0A was revealed in $29.0 \%$ of women. The highest level of knee $0 \mathrm{~A}$ in obese women aged $70-79$ years $-45.8 \%$. According to a chi-squared (X2) test, a significantly higher level of BMI was detected in postmenopausal women with $0 A(X 2=5.05, p=0.02)$.

Conclusion: Women with a symptomatic OA had a significantly higher BMD of lumbar spine compared with women who had a normal functional activity of knee. Significant negative correlation were detected between TBS and BMI, and significant positive correlations between lumbar spine BMD and BMI.
\end{abstract}

KEYWORDS: bone mineral density (BMD), osteoporosis (OP), obesity, postmenopausal women, knee osteoarthritis (OA)

Wiad Lek. 2020;73(3):529-533

\section{INTRODUCTION}

Musculoskeletal disorders (MSDs) are the leading chronic condition that results in deterioration the health level of workforces among European population. Data released by the Ministry of Health of Ukraine suggest that MSDs widely spread in our country among the working age group. The total amount of people suffers from MSDs in Ukraine are up to 4000,000 , and near 2000,000 among them are aged $35-60$ years old [1]. The most prevalent MSDs are osteoarthritis (OA) and osteoporosis $(\mathrm{OP})$ the number of which increases with age.

However, in a case obesity adjunction, the course of any disease worsens. The amount of people with overweight and obesity has highly increased and has nearly tripled since the last decades of previous century $[2,3]$. Nowadays, all over the world, are being conducted an important discussion about OA and OP that is focused on whether being overweight and obese may have a detrimental or protective effect on skeletal health [4]. On the one hand, some studies indicates inverse relationship between OA and OP, as opposite to this the increased bone mineral density (BMD) in patients with $\mathrm{OA}$ does not resulting in reducing risk of osteoporotic fractures [5]. At the same time some studies concludes that obesity has protective role on bone health, while others shows its detrimental effects [6].

\section{THE AIM}

The aim of the study was to investigate the relationship between BMD of lumbar spine, femoral neck, TBS and body mass index (BMI), in postmenopausal women with knee OA and without.

\section{MATERIALS AND METHODS}

The study was performed in D. F. Chebotarev Institute of gerontology, NAMS of Ukraine in accordance with the cooperation agreement between D. F. Chebotarev Institute of gerontology, NAMS of Ukraine and P. L. Shupyk National Medical Academy of Postgraduate Education (NMAPE) from 15 April 2019. The study was approved by the Ethical Committee of NMAPE (05.11.2018, Protocol № 10). All the participants had signed a voluntary informed consent form for participation in research, being the subjects to the respective diagnostic examination procedures.

The study included 359 postmenopausal females aged 50-89 years old, that were divided into four groups by age decades: 87 women aged 50-59 yrs, 162 women aged 60-69 yrs, 88 - aged 70-79 yrs, and 22 women over 80 years old.

The study groups consisted of 117 females with a symptomatic knee OA (I group) and 242 patients without osteoarthritis 


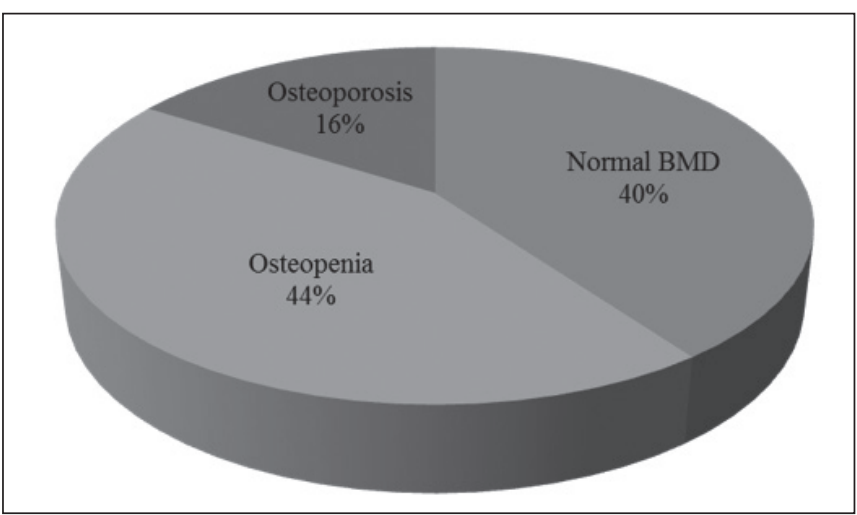

Fig. 1. Distribution of patients according BMD

(II group). Diagnosis of a symptomatic OA was established according to the American College of Rheumatology (ACR) Clinical classification criteria for knee and hip OA (1986). According to these criteria, the presence of knee pain along with at least three of the following six items may be used to diagnose the knee OA: age after 50 years old, morning stiffness of less than 30 minutes, crepitus of knee motion, bone tenderness, bone enlargement, no palpable warmth $[7,8]$. Lower prevalence estimates for a symptomatic rather than a radiographic $\mathrm{OA}$ in the general population reflect the fact that a radiographic $\mathrm{OA}$ is not always accompanied by a clinical disease [9].

The BMD of lumbar spine $\mathrm{L}_{1}-\mathrm{L}_{4}$ and femoral neck was measured by the Dual-energy X-ray absorptiometry (DXA) Hologic (Discovery WI, USA, 2016). TBS of $\mathrm{L}_{1}-\mathrm{L}_{4}$ was detected by TBS insight ${ }^{\oplus}$ software (MedImaps, Pessac, France), which is installed on DXA. Diagnosis osteoporosis was established according to the WHO group described criteria. OP is defined as spinal $\left(\mathrm{L}_{1}-\mathrm{L}_{4}\right)$ or hip (femoral neck) $\mathrm{BMD}$ T-score is equal to or below 2.5 standard deviation (SD) of the young-adult reference mean BMD. Osteopenia is defined as a BMD T-score between- 1.0 and $-2.5 \mathrm{SD}$, and normal BMD - within 1.0 SD.

BMI was computed by the ratio of body weight (kilograms) and height ${ }^{2}$ (meters), expressed in $\mathrm{kg} / \mathrm{m}^{2}$ (WHO,
1998). Diagnosis of obesity was established when BMI was over $30 \mathrm{~kg} / \mathrm{m}^{2}$.

The results are presented in the following manner: Mean values $(\mathrm{M}) \pm \mathrm{SD}$. For data calculation we used: regression, correlation analysis and chi-squared test. A result were considered significant if " $p$ " values were lower than 0.05 $(\mathrm{p}<0.05)$. "Statistika 10.0" (StatSoft, Inc. $\odot$ ) was used for data processing purposes.

\section{RESULTS}

In females with knee OA were detected significantly higher weight and BMI compared with females without OA (tableI). However, there were observed no differences between age and height across all groups.

In this study, no significant differences were found either for group I or II while analyzing the BMD of femoral necks ( $p=0.07$ and $p=0.33$ respectively) and TBS $(p=0.06)$. However, the women with OA had a significantly higher BMD of lumbar spine compared with woman who had a normal functional activity of knee $(\mathrm{p}=0.000068)$ (Table II).

With DXA-based BMD measurement of observed individuals, OP was detected in 54 women (16\% of patients). In 158 females was osteopenia and in 144 was normal BMD (Fig.1).

In both groups there were observed no significant correlation between BMD of L1-L4, hip and age (Fig. 2) At the same time significant negative correlation were detected between TBS and BMI, therefore in a patients with higher BMI, TBS decreases (Fig.3). Correlation and regression analyses between BMI and BMD lumbar spine also shows significant positive relations, what means that at the same time with increasing of BMI increases BMD of lumbar spine (Fig.4).

In the obese subjects, OA was detected in 44 postmenopausal women $(41.1 \%)$, while a normal functional activity of knee - in 63 women (58.9\%). However, out of 73 women with a normal BMI, $29.0 \%$ of cases had a symptomatic OA.

In $42.9 \%$ of women with obesity aged $50-59$ years was detected knee OA comparing with $57.1 \%$ of women without OA. In the group of 60-69 years with obesity, there were

Table I. Anthropometric characteristic of examined women

\begin{tabular}{ccccc}
\hline Parameters & Group I & Group II & F & P \\
\hline Age, years & $65.4 \pm 8.41$ & $65.8 \pm 7.80$ & 0.24 & 0.62 \\
\hline Weight, $\mathrm{kg}$ & $75.3 \pm 15.30$ & $72.1 \pm 12.68$ & 4.39 & 0.04 \\
\hline Height, $\mathrm{cm}$ & $161.9 \pm 6.38$ & $162.2 \pm 6.90$ & 0.20 & 0.65 \\
\hline $\mathrm{BMl}, \mathrm{kg} / \mathrm{m}^{2}$ & $28.8 \pm 5.68$ & $27.4 \pm 4.87$ & 6.45 & 0.01 \\
\hline
\end{tabular}

Note. Group I - postmenopausal women with knee $0 \mathrm{~A}$, Group II - postmenopausal women without $0 \mathrm{~A}$.

Table II. Bone mineral density and trabecular bone score in examined patients

\begin{tabular}{ccccc}
\hline Parameters & Group I & Group II & F & P \\
\hline TBS & $1.24 \pm 0.11$ & $1.22 \pm 0.10$ & 3.32 & 0.06 \\
\hline BMD of lumbar spine & $0.90 \pm 0.16$ & $0.83 \pm 0.01$ & 16.3 & 0.00 \\
\hline BMD of right femoral neck & $0.67 \pm 0.12$ & $0.65 \pm 0.11$ & 3.12 & 0.07 \\
\hline BMD of left femoral neck & $0.66 \pm 0.12$ & $0.65 \pm 0.10$ & 0.91 & 0.33 \\
\hline
\end{tabular}

Note. Group I - postmenopausal women with knee OA, Group II - postmenopausal women without OA. 
detected $39.3 \%$ of patients with symptomatic OA comparing with $60.7 \%$ of females with a normal functional activity of knee. In the group of obese women of 70-79 years, knee OA was revealed in $45.8 \%$ and normal functional activity of knee in $54.2 \%$ of participants. In the oldest group of subjects over 80 years, the OA was in $33.3 \%$ comparing with $66.7 \%$ of woman with a normal functional activity of knee and obesity.

Among women with a normal BMI aged 50-59 years old knee OA was detected in $31.8 \%$ of cases comparing with $68.2 \%$ of those with a normal functional activity of knee joint. In group of 60-69 years, OA was detected in $27.2 \%$ of women comparing with $72.6 \%$ of those without OA. In the group of 70-79 years, there were $25.0 \%$ cases with symptomatic $\mathrm{OA}$ and normal functional activity of knee joint was revealed in $75.0 \%$ of women. In the oldest group of subjects over 80 years, the distribution was $43.8 \%$ of patients knee OA comparing with $56.2 \%$ of women with a normal functional activity of knee joints.

According to chi-squared $(\chi 2)$ test, a significantly higher level of BMI, or more precisely, obesity, was detected in postmenopausal women with knee OA $(\chi 2=5.05, \mathrm{p}=0.02)$. In the first group, there were 44 women with obesity and 73 with normal body weight. In the second group - 63 subjects had obesity and 179 - a normal BMI. In the group of 50-59 year-old women, the values were $\chi^{2}=0.86, \mathrm{p}=0.68$, in the group of $60-69$ year-olds the values were: $\chi 2=2.42, p=0.12$, in the group of $70-79$ year-old the values were: $\chi^{2}=3.56, p=0.05$, and in the group of women over 80 years the values were $\chi=20.20, p=0.65$.

\section{DISCUSSION}

According to the data of the WHO, every one in 4 adults in the world has an overweight and every one in 11 - obesity. Such a high rate of body weight-related problems is alarming due to the fact that an overweight or obesity itself is a risk factor for dozens of diseases. It can cause premature disability and death by increasing the risk of cardiometabolic diseases, OA, dementia, depression and some types of cancers [3].

Nowadays, all over the world, are being conducted an important discussion about obesity, $\mathrm{OA}$ and osteoporosis that is focused on whether being overweight and obese may have a detrimental or protective effect on skeletal health [4]. Historically, obesity has been linked to bone health as a protective factor consequently, some researchers suggest that obesity has protective role on bone health, while others have revealed its detrimental effects. Weight loss in miscellaneous populations including pre- and postmenopausal women leads to a loss of total body BMD up to $2.5 \%$ as well as variable losses at regional bone sites $1 \%-13 \%$. According to the literature data, in premenopausal women greater weight loss (average 14\%) during a relatively short period of time (3-4 months) results in significant bone weakening however a modest weight loss over a longer period of time (6 months) results in fewer or no bone loss [6].

The mechanism behind obesity starts with a similar origin of osteoblasts and adipocytes, both being precursors of a mesenchymal stem cell (MSC). Aging alone alters the MSC in the bone marrow by promoting adipogenesis and reducing osteoblastogenesis [10]. Visceral abdominal fat is the most metabolically active and may be associated with a poor quality of bone tissue and lower BMD. The latter one is also associated with a higher frequency of falls, reducing up to $40 \%$ fat free mass composed mostly of skeletal muscle in the elderly [11] and lower bioavailability of vitamin D accumulated and stored in the fat tissue [12].

Besides, obesity affects the bone metabolism through multiple pathways, including an alteration of bone-regulating hormones, androgens-to-estrogens conversion in adipose tissue, lower serum levels of Sex Hormone Binding Globulin (SHBG), increased serum leptin levels, increased insulin growth factor production and hyperinsulinemia, inflammation, oxidative stress, endocannabinoid system $[6,10]$.

All of these mechanisms could be further enhanced by aging that is another topical issue nowadays [10]. Together with an increasing rate of global aging, the number of people with $\mathrm{OA}$ is noticeably rising. OA characterized by the degradation of cartilage in joints and leads to bones rubbing together and results in stiffness, pain, and impaired movement (WHO) [13]. It usually results in decreased mobility and obesity is a major comorbidity in such patients [14]. Worldwide OA is estimated to affect $15 \%$ of people of different ages [13]. The relationship between OA and OP is complex and controversial [5].

Epidemiological studies suggest that in the same patient $\mathrm{OA}$ and OP rarely occur together. In addition, cross-sectional studies have indicated that $\mathrm{OA}$ is associated with an increase in BMD leading to a common assumption that an increased BMD is protective against OA progression [15].

In the Korean National Health and Nutrition Examination Surveys, there were observed 5793 persons with OA. Their lumbar spine BMD was significantly higher than the one of subjects with knee OA. The findings prompted the conclusion about an inverse relationship between $\mathrm{OP}$ and the presence of knee OA; however, there is a non-linear and site-specific association between $\mathrm{OP}$ and the severity of knee OA [16]. In the Rotterdam study, patients with knee OA had a higher BMD, however the incident fracture risk was increased as compared with those without knee OA [5].

Undoubtedly, there is a certain correlation between BMD and severity of OA. There was reported an increased BMD at all sites in subjects with a moderate-severe OA of either hip, increased BMD at femoral neck and lumbar spine in subjects with a milder hip OA [17].

The Surveys of Osteoarthritis Research Society International (OARSI) consider obesity to be a strong risk factor for knee OA that may also increase the rates of hip, hand and spinal OA, and the number of these painful conditions, together with their associated disability and loss of function, will continue to increase [18].

The Framingham study described an example of decreasing the risk of developing knee OA by $50 \%$ in losing weight females, on average 11 pounds ( 1 pound equals $453.59 \mathrm{~g}$ ). The Clifford survey conclude the possibility of development knee OA increases in 1.36 times with every two units of putting on weight (approximately equal to $5 \mathrm{~kg}$ ) [19].

Recent data from the Intensive Diet and Exercise for Arthritis study suggests that non-mechanical risk factors also play a part in OA development. Weight loss in obese subjects with concomitant knee OA may have anti-inflammatory, as well 


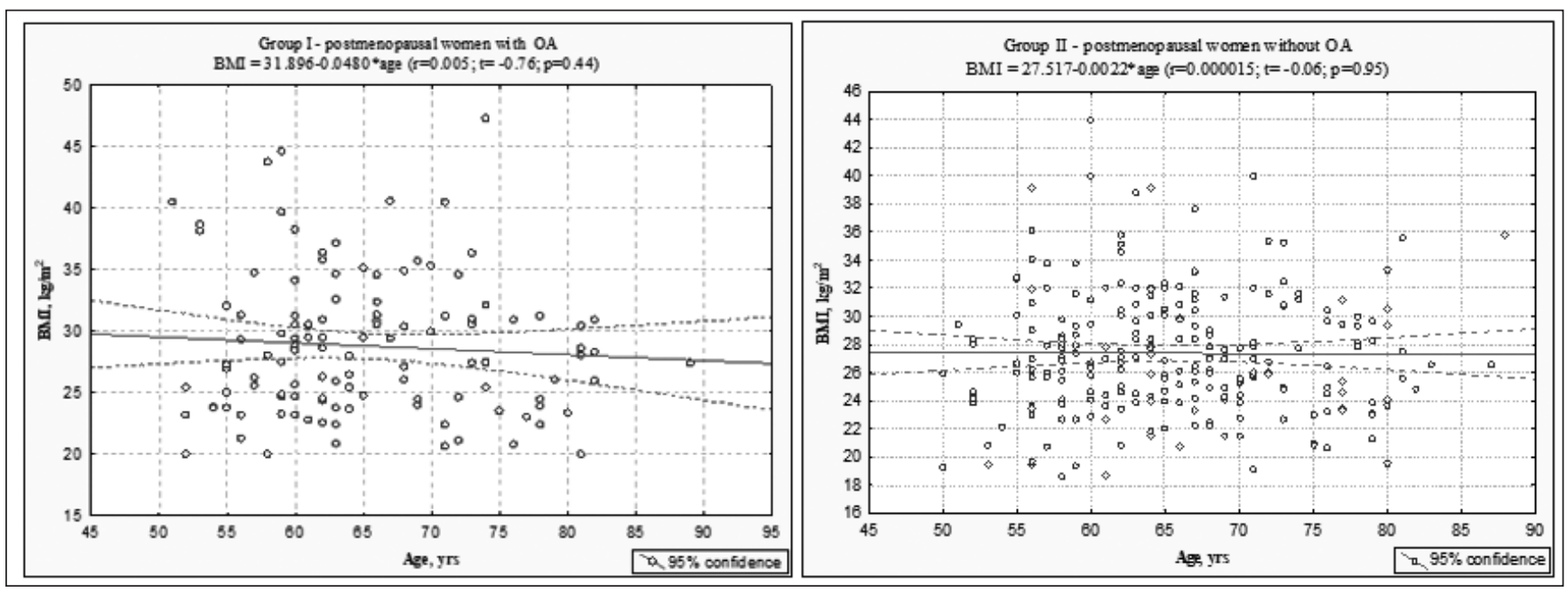

Fig.2. Correlation and regression analyses of relations between $\mathrm{BMI}$ and age in postmenopausal women with and without $\mathrm{OA}$.

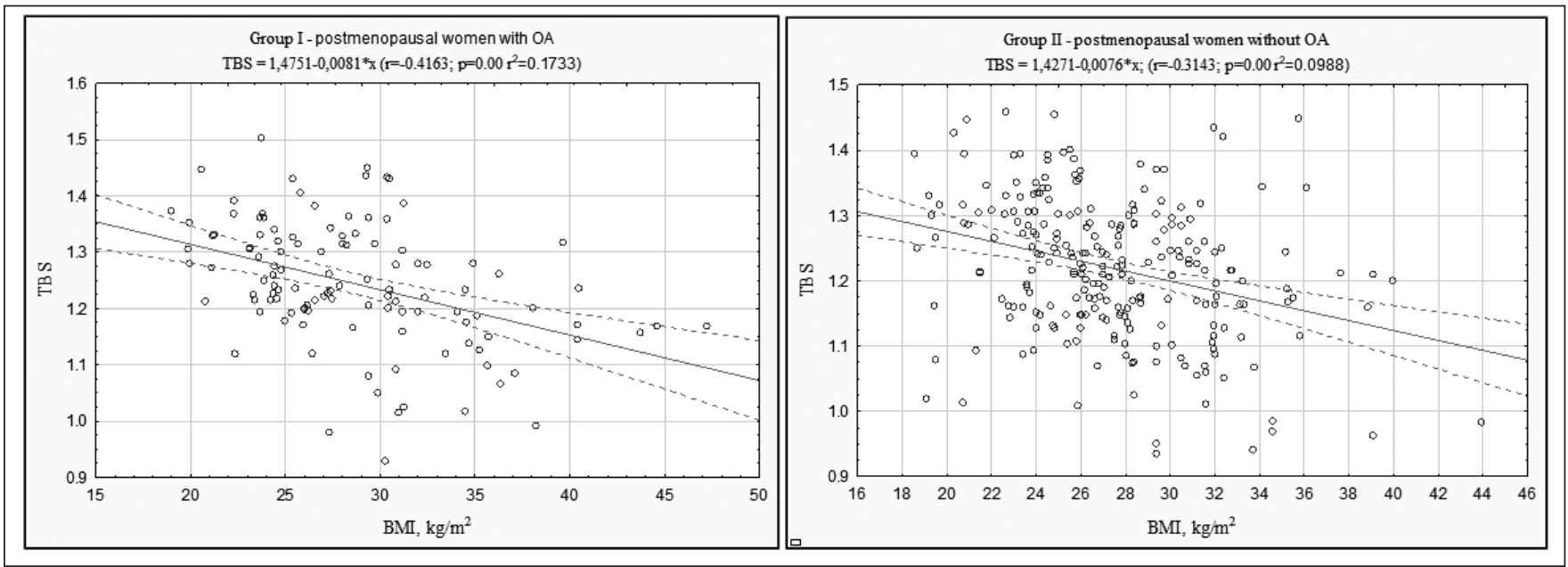

Fig.3. Correlation and regression analyses of relations between BMI and TBS in postmenopausal women with and without OA:
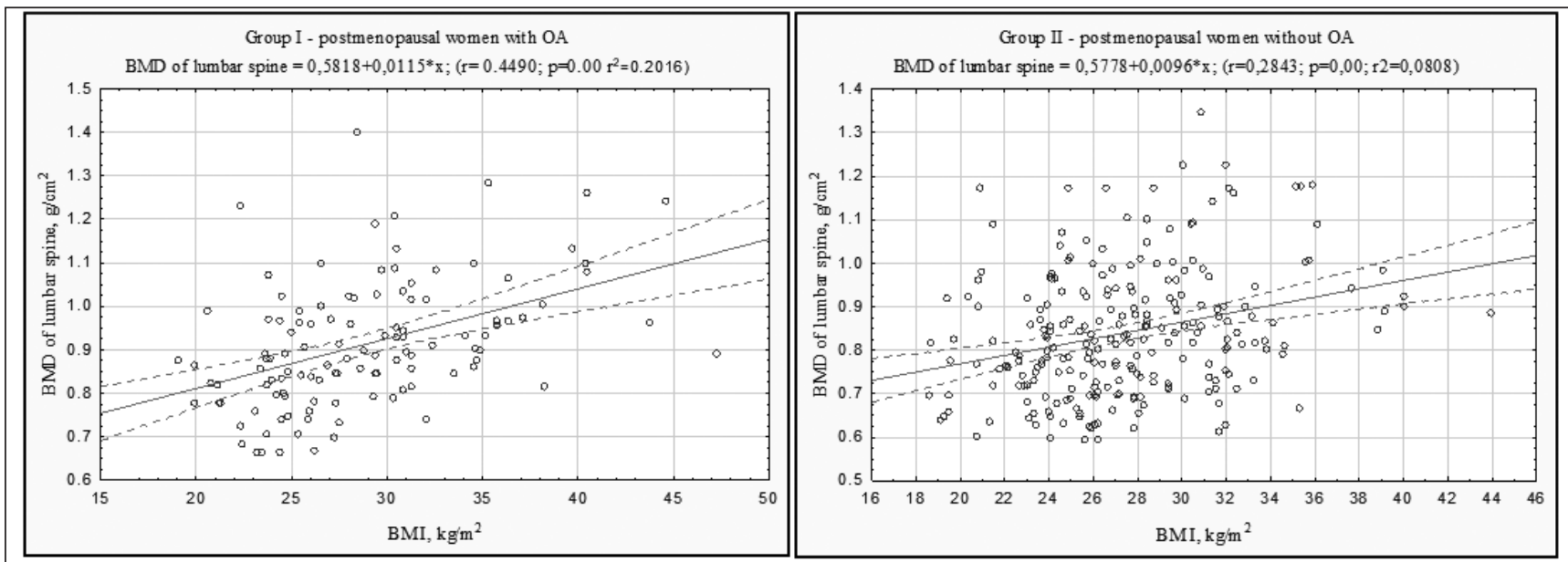

Fig.4. Correlation and regression analyses of relations between BMI and BMD lumbar spine in postmenopausal women with and without OA:

as biomechanical, benefits, that evidenced by reduced IL- 6 levels. Furthermore, beside an association between obesity and OA in weight-bearing joints such as the knee and hip, obesity is also associated with the development of $\mathrm{OA}$ in non-weight-bearing joints, such as those in the hand [20].
This year, a group of Italian scientists was studied TBS in 352 postmenopausal women with obesity. As a result, BMI was found to be negatively related to TBS and positively to the lumbar spine BMD [21,22]. A higher BMD has also been reported in association with OA of the spine [9]. 


\section{CONCLUSION}

The rates of obesity in patients with knee $\mathrm{OA}$, is significantly higher compared to persons with a normal functional activity of knee. Postmenopausal women with knee OA had a significantly higher BMD of lumbar spine compared with women who had a normal functional activity of knee joints. Significant negative correlation were detected between TBS and BMI, and positive correlations between lumbar spine BMD and BMI.

\section{REFERENCES}

1. U.S.-Ukraine Business Council (USUBC). Chamber annual (analytical) publication "Country Profile - Ukraine at a Glance".Washington, D.C. 2014. Available from: https://www.usubc.org/files/Ukraine\%20-\%20 article\%20for\%20country\%20profile\%20-\%20Signed\%200ff\%20 -\%20untracked.pdf

2. World Health Organization. Obesity and overweight. WHO. 2018. Available from: https://www.who.int/news-room/fact-sheets/detail/ obesity-and-overweight

3. Blüher $M$, Obesity: global epidemiology and pathogenesis. Nature Reviews Endocrinology. 2019; 15(5):288-298. doi: https://doi. org/10.1038/s41574-019-0176-8.

4. Salamat MR, Salamat AH, Janghorbani M. Association between Obesity and Bone Mineral Density by Gender and Menopausal Status. Endocrinol Metab (Seoul). 2016;31(4):547-558. doi: 10.3803/EnM.2016.31.4.547.

5. Kim BoY, Kim SS. Incidence of osteoporotic fracture in patients with knee osteoarthritis: clinical impact of frax-based osteoporosis treatment. Ann Rheum Dis: Scientific Abstracts. 2019. 507-508. doi: 10.1136/ annrheumdis-2019-eular.6532.

6. Palermo A, Tuccinardi D, Defeudis G. et.al. BMI and BMD: The Potential Interplay between 0 besity and Bone Fragility. Int. J. Environ. Res. Public Health. 2016; 13(6):544. doi: 10.3390/ijerph13060544.

7. Salehi-Abaril.2016ACR revised criteriafor early diagnosis of kneeosteoarthritis: Autoimmune Diseases an Therapeutic Approaches 2016.3(1): 118-122.

8. Schiphof D, Runhaar J, Waarsing EJ. et. al. The 10-year course of the clinical American college of rheumatology (acr) criteria for hip and knee osteoarthritis in an early symptomatic cohort, data from check. Osteoarthr. and cartilage. 2018;26(1):347-348. doi: https://doi. org/10.1016/j.joca.2018.02.691.

9. Hardcastle SA, Dieppe P, Gregson CL. et.al. Osteoarthritis and bone mineral density: are strong bones bad for joints?. Bonekey Rep. 2015; 4: 624. doi: 10.1038/bonekey.2014.119.

10. Shapses SA, Pop LC, Wang Y. Obesity is a concern for bone health with aging. Nutr Res. 2017; 39:1-13. doi: 10.1016/j.nutres.2016.12.010.

11. McKee A, Morley JE, Feingold KR. et al. Obesity in the Elderly. South Dartmouth (MA):MDText.com, Inc., 2018, Available from: https://www. ncbi.nlm.nih.gov/books/NBK532533/.

12. Biver E. Obesity, fat and bones: friends or foes?. Rev Med Suisse. 2017; 13(559):851-854.

13. Wittenauer R, Smith L, Aden K. World Health Organization. Priority diseases and reasons for inclusion 0steoarthritis 6.12.2013. Availiable from: https:// www.who.int/medicines/areas/priority_medicines/BP6_120steo.pdf

14. Nüesch E, Dieppe P, Reichenbach S, et al. All cause and disease specific mortality in patients with knee or hip osteoarthritis: population based cohort study. BMJ. 2011; 8: 342. doi: 10.1136/bmj.d1165.

15. Yin Z, Wang W, Han J, et.al. Decreased trabecular bone deterioration of proximal tibiae and lumbar vertebrae in postmenopausal women with osteoarthritis. Exp Ther Med. 2017; 13(6):3355-3359. Published online 2017 May 8. doi: 10.3892/etm.2017.4439.
16. Kim YH, Lee JS, Park JH. Association between bone mineral density and knee osteoarthritis in Koreans: the Fourth and Fifth Korea National Health and Nutrition Examination Surveys. Osteoarthritis Cartilage. 2018;26(11):1511-1517. doi: 10.1016/j.joca.2018.07.008.

17. Nevitt MC, Lane NE, ScottJC. et.al. Radiographic osteoarthritis of the hip and bone mineral density. The Study of Osteoporotic Fractures Research Group. Arthritis Rheum.1995; 38(7):907-16.

18. Osteoarthritis: A Serious Disease, Submitted to the U.S. Food and Drug Administration. OARSI. 2016; 1-10. Available from: https://www.oarsi. org/sites/default/files/docs/2016/oarsi_white_paper_0a_serious_ disease_121416_1.pdf.

19. PovoroznyukV, Grigorieva N. Osteoartrozu zhenshchin v postmenopauze: faktory riska i svyaz's kostnoy tkaniu [Osteoarthrosis in postmenopausal women: risk factors and bone connection]. Reproductyvnaia endocrinologyia. 2012, 6(8): 66-70 (in Russian).

20. Bliddal H, Leeds AR, Christensen R. Osteoarthritis, obesity and weight loss: evidence, hypotheses and horizons - a scoping review. Obesity reviews. 2014; 15(7):578-586. doi: 10.1111/obr.12173.

21. Bonaccorsi G, Cafarelli FP, Cervellati C, et al. A new corrective model to evaluate TBS in obese post-menopausal women: a cross-sectional study. Aging Clin Exp Res. 2019. doi: 10.1007/s40520-019-01317-0.

22. Messina C, Buonomenna C, Menon G. et. al. Fat Mass Does Not Increase the Precision Error of Trabecular Bone Score Measurements. J Clin Densitom. 2019; 22(3):359-366. doi: 10.1016/j.jocd.2019.01.001.

The article is a fragment of the scientific research work "Scientific substantiation of modern approaches to optimization of preventive directions at the primary level of providing medical care" (deadline - 2018-2022, state registration number 0113U002455).

\section{ORCID and contributionship:}

Vladyslav V. Povoroznyuk - 0000-0002-8855-482X A,C,F

Nataliia V. Zaverukha - 0000-0002-0181-2794 A,D,E

Anna S. Musiienko - 0000-0002-1672-1991 B,D

\section{Conflict of interest:}

The Authors declare no conflict of interest.

\section{CORRESPONDING AUTHOR Nataliia V. Zaverukha \\ Shupyk National Medical Academy of Postgraduate Education 9 Dorohozhytska str., 04112 Kyiv, Ukraine \\ tel: +38 (067) 5983691 \\ e-mail: nataliyahryb@gmail.com}

Received: 17.01 .2020

Accepted: 05.03 .2020

\footnotetext{
A - Work concept and design, B - Data collection and analysis, C - Responsibility for statistical analysis, D-Writing the article, $\mathbf{E}$-Critical review, $\mathbf{F}$ - Final approval of the article
} 